\title{
¿UN EJERCICIO DE APLICACIÓN DE LA JUSTICIA INTERCUL- TURAL? EL CASO WAORANI EN LA AMAZONÍA ECUATORIANA.
}

\author{
An exercise in the application of intercultural justice? The Waorani case in the \\ Ecuadorian Amazon
} ROBERTO NARVÁEZ*

Fecha de recepción: 10 de junio de 2016 - Fecha de aprobación: 29 de noviembre de 2016

\section{Resumen}

El contexto constitucional en el Ecuador establece los principios de plurinacionalidad e interculturalidad, y tiene un gran vacío en su implementación, impidiendo una relación igualitaria entre ciudadanos, derechos y responsabilidades en un contexto de diversidad cultural. La dicotomía legal del Estado entre una justicia ordinaria positivista y la ausencia de una legislación que reconozca un derecho basado en las costumbres de los pueblos y nacionalidades indígenas. Así, es pertinente abrir la discusión en torno a la justicia ordinaria y la justicia indígena, colaborando con la construcción de mecanismos de diálogo intercultural y estructurando los alcances y limitaciones de las mismas y que puedan ser útiles al establecimiento de un Estado intercultural. El presente artículo realiza un análisis antropológico comparativo del contexto multicultural e intercultural alrededor del caso de diecisiete waorani procesados por el presunto delito de homicidio de un grupo familiar en aislamiento (Tagaeri - Taromenane); hecho acaecido en la selva amazónica ecuatoriana. Se analizan las bases jurídicas del Estado en cuanto a sus principios constitucionales de multiculturalidad e interculturalidad, y la aplicación de los mismos. En este espacio se discuten las limitaciones para el ejercicio de la plurinacionalidad e interculturalidad en la justicia ordinaria ecuatoriana, y los retos para su ejercicio. Así, se aportará a un debate sobre las categorías de multiculturalismo e interculturalidad en el marco del pluralismo jurídico alrededor del estudio de caso.

Palabras clave: pluralismo jurídico, interculturalidad, peritaje antropológico, pueblos indígenas

\begin{abstract}
The constitutional context in Ecuador establishes the principles of plurinational and intercultural, and has problems in this implementation, which has prevented an equal relationship between citizens, rights and responsibilities, in a context of cultural diversity. The legal dichotomy is between a state with positivist ordinary justice against the absence of legislation that recognized a right based on the customs of the indigenous peoples and nationalities. It is pertinent to open the discussion about the ordinary justice and indigenous justice, contributing to the construction of mechanisms of intercultural dialogue and structuring the scope and limitations of them and that may be useful to establish an intercultural state, with regard to justice. This paper makes an anthropological comparative analysis of the multicultural and intercultural context around the case of seventeen Waorani prosecuted for the alleged crime of homicide of a family group in isolation (Tagaeri - Taromenane); in the Ecuadorian Amazon rainforest. It analyzes the legal bases of the state in terms of its constitutional principles of multiculturalism and interculturality, and the application of them. This space discusses the limitations for the exercise of plurinationality and interculturality in the ordinary Ecuadorian justice, and the challenges for its exercise. Thus, it will contribute to a debate on the categories of multiculturalism and interculturality within the framework of legal pluralism around the case study.
\end{abstract}

Keywords: legal pluralism, intercultural, anthropological expertise, indigenous peoples

* Licenciado en Antropología de la Pontificia Universidad Católica del Ecuador. Investigador independiente. Quito, Ecuador. Correo-e: robertonarvaezc@gmail.com 


\section{Introducción}

La realidad histórica de Latinoamérica se ha caracterizado por la coexistencia de pueblos y nacionalidades indígenas y sociedades blanco-mestizas, en un espacio donde confluyen las estructuras del Estado (Gazohui, 2008), las formas de gobierno y el territorio. En el Ecuador, desde 1998 la Constitución incluyó los principios de multietnicidad y pluriculturalidad, como un reconocimiento de la diversidad cultural del país. La Constitución desarrollada y aprobada en 2008 integró los principios de plurinacionalidad e interculturalidad. Pese a lo anterior, la institucionalidad del Estado ecuatoriano evidencia limitaciones en la incorporación de estos principios, que se refleja en el poco desarrollo de políticas públicas plurinacionales e interculturales efectivas al no existir instrumentos procesales que viabilicen la ejecución y práctica real de estos derechos.

El reconocimiento a la diversidad étnica y cultural también encuentra sus límites en el ámbito jurídico, con un conflicto entre la prevalencia de la justicia ordinaria que propugna la consideración de igualdad de los miembros del Estado frente a la existencia de sistemas de justicia consuetudinarios. Lo anterior, limita el acceso y la aplicación real de la justicia en un escenario multicultural y demuestra cómo los principios constitucionales de plurinacionalidad e interculturalidad tienen un vacío en su aplicación, al reafirmar las relaciones de poder que subordinan a los pueblos indígenas (Stavenhagen, 2000a) a un Estado hegemónico blanco-mestizo. Además del vacío procedimental, el desenvolvimiento entre la justicia ordinaria y la justicia indígena requiere de la interpretación de los casos, y es allí en donde la capacidad para el entendimiento de la información y los hechos desde el ámbito cultural recae sobre la ciencia antropológica profesional. Este espacio para la participación de la antropología en el sistema judicial de un país debe ser diseñado y construido en las instituciones judiciales del Estado, con el fin de que se convierta en un "elemento de comunicabilidad entre la justicia indígena y la justicia estatal, entre el derecho propio de los pueblos y nacionalidades indígenas, y el derecho blanco - mestizo" (Ávila, 2011: 16).

En el Ecuador y en relación a la aplicación de la justicia, la dicotomía legal no se da entre un país con tradición en el derecho positivo occidental frente a un derecho consuetudinario perteneciente a los pueblos y nacionalidades indígenas, sino entre el Estado con justicia ordinaria positivista frente a la ausencia de una legislación que reconozca un derecho basado en los usos y costumbres de los pueblos y nacionalidades indígenas; tal es el caso del Código Orgánico de la Función Judicial (Asamblea Nacional del Ecuador, 2009) que se limita a establecer procedimientos interculturales bajo los principios de diversidad, igualdad, Non bis in ídem, pro jurisdicción indígena e interpretación intercultural, y la declinación de la competencia de la justicia indígena. Así, nuevamente se evidencia la condición de subordinación de estos últimos en los Estados de las Américas, donde han sido invisibilizados y han ocupado un lugar secundario y al margen de los Estados ${ }^{1}$ (Díaz Polanco, 1981; Stavenhagen, 2000b; Clavero, 2008). De la misma manera, la Ley Orgánica de Garantías Jurisdiccionales y Control Constitucional (Asamblea Nacional del Ecuador, 2009) establece una serie de garantías frente a decisiones de la justicia indígena. Es justamente en este ámbito, en donde la práctica antropológica profesional requiere hacer su mayor aporte ya 
sea alimentando los espacios de discusión en torno a la justicia ordinaria y la justicia indígena, colaborando a la construcción de mecanismos de diálogo intercultural, desarrollando propuestas más allá de un discurso sobre el tema, y además, estructurando los alcances y limitaciones de las mismas y que puedan ser útiles al establecimiento de un Estado intercultural, en lo que se refiere a la justicia.

El presente texto expone un análisis antropológico de orden comparativo, del contexto multicultural e intercultural con el cual se abordó judicialmente el caso de diecisiete waorani ${ }^{2}$ procesados por el presunto delito de homicidio de un grupo familiar Tagaeri - Taromenane ${ }^{3}$ de pueblos en aislamiento en el Yasuní, amazonía ecuatoriana quienes provienen del mismo tronco cultural y lingüístico. Partimos de cuestionar si las bases jurídicas del Estado ejercen los principios constitucionales de multiculturalidad e interculturalidad, y si es así, cuál es el grado de aplicación de dichos principios. Adicionalmente, nos interrogamos si desde la práctica antropológica se aporta o no a la implementación de la justicia en el marco de la interculturalidad. Para contestar las interrogantes expuestas anteriormente, nos planteamos discutir conceptualmente, desde el enfoque de la antropología con énfasis en lo jurídico, las limitaciones para el ejercicio de la plurinacionalidad e interculturalidad en la justicia ordinaria ecuatoriana, además de determinar los retos para su ejercicio. Así, buscamos aportar a un debate sobre las categorías de multiculturalismo e interculturalidad en el marco del pluralismo jurídico alrededor del estudio de caso.

Iniciaremos con una exposición breve del estudio de caso, para continuar con la discusión sobre el contexto constitucional de la justi- cia tradicional en el Ecuador identificando los paradigmas que construyeron este marco legal. A continuación, se analizarán los límites de la justicia consuetudinaria desde la visión de la antropología jurídica para plantear los retos que existen en el ejercicio de una justicia plural en el Ecuador. En el último capítulo, se estudiará el caso judicial seleccionado enfocándose en el proceso legal que se llevó a cabo, los hechos culturales implicados en el mismo, los límites de la justicia ordinaria y los desafíos para el establecimiento y construcción de una justicia intercultural en el Ecuador.

\section{Antecedentes}

En mayo de 2003, la noticia de una masacre múltiple ocurrida en un lugar recóndito y poco conocido de la Amazonía ecuatoriana estremeció al país. El hecho suscitado en contra de algunos miembros pertenecientes a Pueblos Indígenas en Aislamiento (PIA) ${ }^{4}$ localizados en el sector del Yasuní, encendió las alertas sobre las actividades extractivas que suceden en esta zona y que amenazan a pueblos vulnerables. El lugar, Tihuino, un poblado waorani, es conocido por tener una larga historia de conflictos entre pueblos indígenas y grupos mestizos relacionados principalmente con la extracción de recursos naturales, también es notable por una ausencia del Estado, y donde priman las relaciones con actores externos como las empresas petroleras y otros relacionados con el turismo. Desde hace más de treinta años, en este sector amazónico se explota petróleo, sin embargo, a partir de 1999, el líder del poblado waorani permitió a través de acuerdos con actores externos que la actividad de extracción maderera se realice desde el interior de un territorio virgen, 
la Zona Intangible Tagaeri Taromenane ${ }^{5}$. Este espacio delimitado por las autoridades ecuatorianas para el tránsito y la protección de los pueblos en aislamiento Tagaeri y Taromenane, ha sido violentado en varias ocasiones y por distintos grupos interesados (Aguirre, 2007). Se presume que una de las principales causas para el ataque contra los Tagaeri fue de índole cultural, para vengar la muerte de un poblador waorani de Tihuino quien murió en manos de Tagaeri en 1996. Si bien los grupos familiares en aislamiento en el Yasuní tienen filiación cultural y lingüística waorani, por el orden social existente en los waorani se mantienen conflictos que permanecen latentes aún después de varios años. También se presume existía el interés del líder waorani por asegurar los ingresos económicos que percibían por la explotación maderera en el territorio de uso y movilidad tradicional de los PIA.

En base a las circunstancias relatadas y en el contexto legal antes expuesto, es evidente que no existió una verdadera investigación judicial que pudiera determinar todos los componentes culturales que intervinieron en este hecho y a la vez, pudiese establecer las responsabilidades que cada uno de los grupos involucrados generó frente a un delito repudiable por la sociedad en general regida por el derecho ordinario.

Por el contrario, lo único que se puede encontrar al respecto es una investigación inconclusa a cargo de la Fiscalía de Pastaza, que tuvo una fuerte presión de parte de la organización waorani $\left(\mathrm{ONHAE}^{6}\right)$ y otras organizaciones indígenas del país ${ }^{7}$, a fin de que se considere al ataque, y masacre, como una acción enmarcada en la cultura y por tanto sujeta al ámbito consuetudinario, a pesar de que los hechos implicaron otros factores que no fueron consi- derados en el análisis, limitándose a una posición esencialista que consolidó el pedido de impunidad de los atacantes. Si bien se realizó la diligencia fiscal de reconocimiento del lugar de los hechos y se encontraron los cuerpos de al menos veinticinco personas asesinadas con armas de fuego y lanzas (Vargas \& Gálvez, 2007), el Fiscal a cargo se abstuvo de iniciar la Instrucción Fiscal y recurrió a formalismos como la ausencia de documentos de identidad de las víctimas. De esta manera, la masacre no llegó ni siquiera a constituirse en un proceso de instrucción fiscal ni de formulación de cargos, y así el caso fue cerrado dejando el hecho en la impunidad.

Para el Ecuador, este hecho refleja no solo el desconocimiento de otras formas de vida y de otras realidades que conviven y se albergan en el país, sino que, además, devela la incomprensión que existe a la otredad y a la diversidad cultural por parte de una de las instancias principales en lo que se refiere la estructura del Estado, el poder judicial. Como se mencionó, la ONHAE a través de sus dirigentes ${ }^{8}$ exigió que no se desarrolle ningún proceso judicial en relación a este caso en el ámbito ordinario, y solicitó la competencia de la justicia indígena9 Esta acción supuso el ocultamiento de los involucrados y su defensa velada en contra de cualquier tipo de acción legal en su contra. La organización waorani argumentó "motivos tribales" (Rivas, 2003) como justificación al ataque, remitiéndolo a un hecho cultural de venganza y limitando el tema a un discurso esencialista y culturalista que buscaba justificar la acción, en el hecho de la pertenencia étnica de los autores y su condición de pueblo indígena.

Así, las consecuencias que se identificaron desde el dato etnográfico fueron dos: la primera, 
no existió el análisis judicial respectivo, lo que significa que no se desarrolló jurisprudencia; la segunda, se motivó un sistema de impunidad que incidió en hechos posteriores. La conmoción social que despertó esta masacre en la opinión pública ecuatoriana no tardó en desvanecerse, sin permitir que el Estado y la sociedad nacional en su conjunto pudieran discutir y cuestionar sobre la necesidad de contar con procesos judiciales diversos que impidan la impunidad, sobre todo en casos en los que factores culturales se encontraran involucrados.

A pesar de que la Constitución de 1998, vigente hasta la fecha de ocurrido el suceso, ya definía al Estado como pluricultural y multiétnico en una versión culturalista que dio respuesta a la lucha y a varias de las reivindicaciones planteadas por las organizaciones indígenas, en el ámbito de la justicia no se establecía ningún procedimiento para la coordinación entre la justicia ordinaria y la indígena, por lo que la justicia ordinaria debía hacerse cargo del proceso judicial. Por ello, no se consideraron las características culturales de las víctimas ni de los atacantes, quedando el hecho en la impunidad. De esta manera, el sistema judicial ecuatoriano perdió la oportunidad de resolver el caso, en la medida en que no tenía la estructura para hacerlo, y perdió la posibilidad de crear jurisprudencia al respecto. Frente a esto, un análisis constitucional realizado por Grijalva (2008) menciona que los alcances de la Constitución de 1998 en materia de justicia indígena se limitaron a "lo nominal, a una suerte de retórica constitucional inoperante o meramente legitimadora frente a las instituciones y la sociedad" (50).

El 30 de marzo de 2013, se dio un nuevo hecho que afectó a pueblos en aislamiento y ocasionó la muerte de varios de sus miembros: fue reali- zado por un grupo de pobladores waorani de varios poblados (Yarentaro, Dicaro, Guiyero y Tihueno), quienes ingresaron al territorio y generaron un ataque contra los pueblos Tagaeri - Taromenane. El hecho fue una venganza por la muerte de dos ancianos waorani, Ompore Omehuai y su esposa Buganei Caiga, sucedida un mes atrás. Los dos ancianos en mención fueron lanceados por un grupo familiar de PIA; por estas muertes, los familiares de las víctimas organizaron el ingreso al territorio, buscando ejecutar la venganza por las mencionadas pérdidas. Se estima que al grupo de atacantes waorani le tomó varios días de caminata por la zona hasta llegar a una casa de una familia en aislamiento, misma que fue atacada.

En este nuevo caso, en la etapa de indagación previa e instrucción fiscal se realizaron estudios e investigaciones antropológicas para conocer detalles sobre el hecho, los mismos que fueron alimentados por las entrevistas a los atacantes, a su entorno familiar, social y cultural; además, existen versiones de los atacantes, quienes inicialmente señalaban la muerte de más de veinte individuos, entre adultos y niños. Entre los estudios, se realizaron pericias antropológicas y la recolección de información de elementos culturales en la diligencia fiscal de reconocimiento del lugar donde se suscitaron los hechos. De esta manera, y a diferencia de lo que sucedió en el 2003, la Fiscalía General del Estado avanzó con el proceso encaminándose en los lineamientos establecidos por la Constitución en lo que se refiere a la interculturalidad, respetando la normativa establecida en el Código Orgánico de la Función Judicial, el Código Penal (vigente para el presente caso) y posteriormente, la aplicación del Código Integral Penal específicamente a lo que se refiere el respeto a la diversidad cultural del país. 
A pesar de las diferencias con los hechos de 2003 y el proceso que siguió la Fiscalía en este caso, la complejidad del proceso judicial radicó en varias circunstancias relacionadas con vacíos procedimentales y de jurisprudencia. Una vez concluida la fase de indagación previa, se dio inicio a la etapa de instrucción fiscal y dentro de esta, el juez a cargo del caso y a pedido de la Fiscalía, realizó una Consulta a la Corte Constitucional con la finalidad de que "se resuelva sobre la constitucionalidad de la norma contenida en el artículo 1 de la Ley s/n Registro Oficial 578-S del 27 de abril de 2009, inserto antes del artículo 441 del Código Penal, en el que se encuentra prevista la sanción por la comisión del delito de genocidio"10, denotando, nuevamente, una ausencia de elementos culturales y de cosmovisión para una interpretación de la Constitución y las leyes desde una perspectiva intercultural.

\section{Multiculturalismo e interculturalidad en la justicia ordinaria en Ecuador}

El estudio de caso presentado en este trabajo investigativo permite analizar las restricciones existentes en la implementación de los conceptos y prácticas del multiculturalismo y la interculturalidad en el sistema de justicia ecuatoriano. A continuación, plantearemos algunas de las limitaciones y retos que se han identificado en esta problemática.

\subsection{Limitaciones: el paradigma del multicul- turalismo en la justicia del Ecuador}

Tras los hechos sucedidos en 2003 en torno a la masacre ocurrida entre miembros de clanes indígenas distintos, el caso en mención permite identificar la contradicción jurídica que existe en la normativa ecuatoriana, cuando desde un contexto constitucional, la diversidad étnica ha sido reconocida mientras, por otro lado, el tema de la justicia indígena no ha sido considerado en otros cuerpos legales secundarios ni procedimentales, limitando así el análisis cultural requerido para la solución de estos casos en el marco de un Estado pluriétnico y multicultural.

En el ejemplo jurídico citado, la Constitución ecuatoriana se redujo a reconocer las distintas prácticas de justicia vigentes en los pueblos indígenas" (Cervone, 2009), sin que sus elementos culturales constitutivos y de saber consuetudinario, lleguen a alimentar al sistema nacional de justicia. Por esto, en el Ecuador varios procesos legales han requerido una amplitud en su tratamiento, sin embargo, no han logrado ser abordados desde su particularidad a fin de generar sentencias que se encuentren apegadas a los lineamientos constitucionales, es decir, al ejercicio pleno de los derechos de las nacionalidades y pueblos originarios.

Adicionalmente, es posible evidenciar cómo esta práctica homogénea de lo legal entra en contradicción con otras normativas internacionales suscritas por el país en mención, al no garantizar el respeto a los derechos colectivos de los pueblos indígenas establecidos en el Convenio 169 de la OIT, que establece consideraciones en relación a las costumbres o derecho consuetudinario, reconocimiento a sus autoridades, a sus características económicas, sociales, culturales, y sanciones diferenciadas a la prisión, entre otras.

En el caso del 2003, lo que menos se consideró en este ámbito fueron las características culturales de las víctimas, al punto de no proce- 
der la instrucción fiscal por no existir documentos de identidad de las mismas, pese a contar como evidencia los cuerpos de los fallecidos. Estas circunstancias fueron algunas de las razones por las que la masacre quedó en la impunidad. En el entorno institucional y judicial, el Defensor del Pueblo de Pastaza de ese entonces, solicitó a los operadores de justicia a cargo del caso que consideren los derechos colectivos de los atacantes enfatizando la importancia de dos normas constitucionales específicas: el artículo 84 que hacía referencia a: "conservar y desarrollar las formas tradicionales de convivencia social y ejercicio de la autoridad", y el artículo 191 que consagraba el derecho consuetudinario "en la solución de sus conflictos". La solicitud realizada por dicha autoridad, ni siquiera llegó a discutirse en este caso, simplemente, porque el proceso se suspendió y con él, cualquier posibilidad de justicia.

Por su parte, desde los dirigentes de la nacionalidad waorani, se tomó una "decisión judicial" respecto al caso. En esta, los expresidentes de la organización junto a algunos ancianos y los mismos atacantes, decidieron el indulto hacia los agresores, ejerciendo así una actuación de "justicia indígena" que no se amparaba en ningún sistema de justicia indígena waorani y no se enmarcaba en sus formas culturales tradicionales de resolución de conflictos, ni en el derecho consuetudinario. Los líderes waorani de la ONHAE, la dirigencia de la Confederación de Nacionalidades Indígenas del Ecuador (CONAIE), y la organización regional amazónica a través del Consejo de Organizaciones Indígenas de la Cuenca Amazónica (COICA) (Chávez, 2003), hicieron un uso interesado del multiculturalismo, al plantear un escenario en el cual las autoridades y ancianos waorani debían establecer la sanción correspondiente, enmar- cados en sus "tradiciones" (Rivas, 2003), sin considerar los derechos de las víctimas.

Frente a esta situación, es importante señalar que cuando existen diferentes actores involucrados, como en este caso fueron las organizaciones indígenas, las discusiones y las resoluciones pueden tornarse bastante complejas y poco objetivas. De esta manera, fue como estos colectivos no plantearon una justicia indígena sino un relativismo cultural bajo el precepto de que no existían criterios universales para juzgar las acciones particulares de un pueblo, y así recogieron y de manera intencionada, ciertos preceptos del multiculturalismo que tal como expone Velasco Gómez (2004), representan un riesgo porque nos ubican en un relativismo extremo cercano a un paternalismo e incluso a un etnocentrismo.

Cervone (2009) manifiesta que, en Ecuador, a partir de la Constitución de 1998, la justicia indígena o los sistemas de derecho consuetudinario no han sido reconocidos a plenitud y solo se ha permitido su ejercicio en los casos en los cuales no se oponen a los principios del sistema nacional de justicia. Es decir, se ha mantenido una jerarquía en relación a la prevalencia del derecho positivista del sistema judicial ordinario, por sobre los sistemas de justicia indígena, por esto, el pluralismo ha sido dependiente de que su desarrollo y resoluciones no sean contrarios a lo establecido en la justicia ordinaria. Otra especialista relacionada al tema de justicia e interculturalidad, Sánchez Botero (2010), señala que las contradicciones o incompatibilidades, esto es, los aspectos que no pueden ser incorporados por los sistemas de justicia positivista, "son resueltos por la vía de la imposición de la soberanía nacional del Estado y de su correspondiente derecho" (Op.cit.: 147). 
Lo cual deja entrever cómo la aplicación de la justicia en el Ecuador no ha encontrado los caminos adecuados para hacer que sus leyes tengan relación entre todos los cuerpos legales existentes y garanticen los derechos de las minorías, en este caso, étnicas.

Con lo anterior, se evidencia que el Estado ecuatoriano a pesar de los avances en el derecho constitucional, sigue siendo predominantemente unicultural, ya que no se ha traducido la norma y enunciados constitucionales en leyes ni en reglamentos que permitan el ejercicio real de la plurinacionalidad y de la interculturalidad, lo cual se ahonda si se considera que tampoco existe jurisprudencia que se exprese en una sentencia intercultural, y por eso no hay un avance efectivo en la protección de estos derechos, principalmente de las víctimas.

Es evidente que estos vacíos legales no permiten la práctica de una legislación oportuna en estos temas jurídicos-culturales, ya que no se abre ni se agiliza la posibilidad de sancionar un hecho cometido por miembros de un pueblo o nacionalidad indígena bajo su cosmovisión, debido a que el operador de justicia no tiene la capacidad de emitir una sentencia y crear jurisprudencia, exhibiendo así la ausencia de una política pública que permita la actuación real del pluralismo jurídico.

De la misma manera y a la vez, desde otra perspectiva, el campo de acción y de jurisdicción de la justicia indígena en el Ecuador se ha limitado a espacios comunitarios de las nacionalidades y pueblos originarios, aislándolos de otros escenarios más amplios dentro de la política nacional y del sistema ordinario de justicia, tal como se ha demostrado en la sentencia de la Corte Constitucional. Precisamente, el caso que presentamos en este trabajo ha sido el que mayormente ha trascendido a la esfera pública y a la demanda de una decisión político-jurídica $^{11}$ que permita identificar y analizar varios aspectos que guíen los lineamientos a través de los cuales sea posible el ejercicio de la plurinacionalidad e interculturalidad y los derechos de los pueblos indígenas. En el desenvolvimiento judicial de este caso, el procedimiento de índole intercultural fue asumido desde las primeras acciones de la justicia ordinaria y en relación al desarrollo del proceso de indagación previa impulsado desde la Fiscalía. Por ejemplo, se realizaron diversas diligencias como la toma de versiones a los presuntos atacantes, a testigos, y a personas de la comunidad de donde proceden los presuntos implicados. Los waorani involucrados contaron con defensores públicos y particulares, y se empleó su lengua nativa, wao tededo para la toma de declaraciones. Adicionalmente, se contó con traductores de la lengua wao al castellano, por lo que, las versiones fueron recogidas desde la particularidad lingüística de los presuntos atacantes y el idioma oficial que rige al sistema ordinario de justicia; así mismo, se procedió a una interpretación intercultural del caso, a través de peritajes culturales y sociales para la reconstrucción de los hechos.

En el proceso de indagación previa hasta la etapa de instrucción fiscal las consideraciones de interculturalidad fueron pieza clave; sin embargo, en relación a la plurinacionalidad, es decir, al reconocimiento y ejercicio de la justicia indígena, no existieron espacios donde se planteara la incorporación del derecho consuetudinario en este caso. De igual manera, tampoco existió solicitud alguna de declinación de competencia de la justicia ordinaria en favor de la indígena, y esto tuvo relación con la sentencia que emitió la Corte Constitucional en el caso 
denominado "La Cocha" (Sentencia № 113-14SEP-CC, 2014), en el cual, se limitó el ejercicio de la justicia indígena cuando el derecho vulnerado fuera "el derecho a la vida". En consecuencia, la única jurisdicción aplicable en casos relacionados con el mencionado derecho, es la justicia ordinaria.

Si bien el artículo 171 de la Constitución ecuatoriana del año 2008 , restringe la jurisdicción indígena a la resolución de conflictos internos que no impliquen violación a derechos consagrados en la Constitución y tratados internacionales de derechos humanos, tampoco abrió la discusión a la pertinencia o no de los sistemas de justicia indígena, o a la construcción de un espacio en el que se construya una justicia intercultural.

\subsection{Retos: la interculturalidad en la justicia ecuatoriana}

Las normas y tratados internacionales de derechos humanos vigentes abren la posibilidad de reconocimiento a los derechos de los pueblos y nacionalidades indígenas, e impulsan a los países firmantes a la necesidad de construir formas de resolución de conflictos interculturales, por ello, el reconocimiento de la diversidad entendido como "la coexistencia de sistemas culturales distintos (Díaz Polanco, 2006), y "la necesaria convivencia de varios enfoques doctrinales de carácter comprensivo que permite a los individuos construir distintas visiones del mundo, de los fines de la existencia y concepciones de lo que para cada uno de ellos constituye una vida buena" (Op.cit.: 17), es posible, limitando la imposición del concepto de "pluralismo jurídico formal unitario" (Hoekema, 2002) en el cual el derecho consuetudinario mantiene un papel complementario al derecho ordinario.
En este aspecto, la experiencia colombiana con características históricas y sociales similares al Ecuador, tiene avances significativos en la interpretación de los derechos denominados "propios" de los pueblos indígenas (Sánchez Botero, 2010), que se caracterizan por su jurisdicción local, por "tener reconocimiento social y ordenar pautas de comportamiento, restricciones y prohibiciones", entre otros aspectos, "representan la existencia de otros horizontes que no se fundamentan y estructuran en los principios occidentales" (Op.cit.: 150), esto es en el derecho positivo, o, como lo menciona Hoekema (2002), el derecho oficial reconoce la validez de diversos sistemas de derecho. Es así que la práctica antropológica profesional ha aportado al conocimiento especializado a través de los peritajes antropológicos, gracias a la capacidad de plantear argumentaciones y razones de convencimiento sobre conocimientos, hechos o prácticas culturales involucradas en casos judicializados (Matos, 2010; Valladares, 2011; Masapanta, 2009; García, 2007). Esto ha proporcionado al juez los elementos necesarios para el entendimiento de un objeto o causa específica, aportando pruebas (Valladares, 2012) para demostrar hipótesis, que tienen toda la posibilidad de convertirse en pruebas plenas, cuando son consideradas en las sentencias emitidas por los jueces.

Esta argumentación cultural es un elemento claro y preciso que aporta al proceso de interrelación entre dos actores principales, por una parte, un Estado con un conjunto de leyes y normativas que han sido desarrolladas desde una visión meramente occidental, y los pueblos y nacionalidades indígenas que reproducen una serie de parámetros culturales propios. Los conflictos culturales y normativos legales que se desenvuelven en una nación multicultural, no 
pueden entenderse solo desde la lógica de la normatividad hegemónica, por lo cual, se hace imprescindible fomentar espacios de discusión para que las relaciones interculturales se reconozcan en la diversidad cultural y puedan concebirse, así como las dinámicas culturales particulares que intervengan en conflictos judiciales. Es muy necesario que ciertas prácticas, creencias, cosmovisiones, y particularidades culturales, sean explicadas e interpretadas bajo esquemas y referentes cognitivos de las propias culturas desde donde emergen y donde se manifiestan, con el objeto de significar lo que ellas mismas expresan, creando así un espacio de relacionamiento intercultural en el que se llegue a entender la diversidad que cohabita en un país y su gente.

El camino que plantea la interculturalidad es el de confrontar para transformar las relaciones y estructuras de poder, extendiendo estos debates a la estructura del sistema jurídico (Walsh, 2012), resultado de la colonialidad de poder y la racionalidad occidental de un estado unicultural; es decir, se requieren cambios profundos en las estructuras, caso contrario el reconocimiento a la diversidad de sistemas jurídicos consuetudinarios incorporará solamente elementos que no cuestionen ni amenacen las dinámicas del Estado unicultural. Por ello, Walsh (2012) plantea que la sentencia del Caso № 0072 14-CN (Sentencia № 004-14-SNC-CC, 2014), que corresponde a la consulta de norma impulsada por el Dr. Álvaro Guerrero, juez segundo de garantías penales de Orellana, sobre el tipo penal de genocidio para el caso de los waorani acusados de asesinato, debe apoyar a la construcción de un modelo intercultural en la justicia, rompiendo con cualquier jerarquía de un sistema de justicia sobre otro, evitando así que la interculturalidad se remita a un ámbito funcional.
La diversidad cultural existente en el Ecuador expone una serie de problemáticas en el relacionamiento intercultural, principalmente, desde la institucionalidad del Estado hacia las nacionalidades y pueblos indígenas; esto debido a que aún existen limitaciones en las distintas leyes que permitan un ejercicio pluricultural de la justicia. En la realidad, la incorporación de elementos de la justicia indígena al sistema ordinario no responde a una imposición cultural, ya que esos elementos incorporados serían solamente aquellos que no afecten al esquema constituido desde el derecho positivo. Es por esta situación, que los sistemas de justicia consuetudinarios deben ser ejercidos desde el territorio, dentro de una lógica particular de este espacio territorial.

\section{La construcción de una justicia intercultural}

El contexto conceptual y teórico desarrollado en los apartados precedentes, en referencia al proceso legal por presunto delito de homicidio seguido en contra de diecisiete miembros de la etnia Waorani por afectar a un grupo familiar de los pueblos en aislamiento, ha permitido considerar diferentes elementos desde diversos ámbitos. Por ejemplo, los principios de plurinacionalidad e interculturalidad establecidos en la Constitución de 2008, no han sido suficientes para garantizar el ejercicio mismo de dichos derechos. No solo es preciso el reconocimiento de la diversidad cultural en el Ecuador, sino que este logro debe ir de la mano del desarrollo de políticas públicas que efectivicen estos derechos.

En el ámbito judicial, puntualmente, en el de la justicia indígena, el reconocimiento del Estado a la existencia de otros sistemas legales debiese implicar el reconocimiento a un poder 
otorgado por un grupo social, con sus propias normas, autoridades, procedimientos, sanciones y garantías de un orden social. Este ejercicio dinamizará un pluralismo jurídico. En el Ecuador, los sistemas de justicia indígena han funcionado al margen del orden estatal, y su reconocimiento ha estado limitado a situaciones del ámbito interno de las comunidades, pueblos y nacionalidades indígenas. Es por ello que, en casos particulares relacionados con delitos de carácter penal, la opinión pública ha destapado el conflicto que tiene el Estado y su sistema de justicia ordinario para administrar una justicia que integre a esos otros sistemas de justicia presentes en el país.

La matanza de 2013 y el proceso judicial que se desarrolla en dicho caso, nos permite identificar que el sistema de justicia ordinario tuvo una serie de limitaciones para abordar los principios de plurinacionalidad e interculturalidad establecidos en la Constitución. Una de las posibles limitaciones es el conocimiento y reconocimiento de la diversidad cultural del Ecuador, y la comprensión de las formas organizativas, de ordenamiento social interno, y otras formas tradicionales de convivencia de los waorani. Por otra parte, el sistema ordinario pudo haber generado un espacio de diálogo con el sistema tradicional waorani y discutir su pertinencia de una manera más amplia.

Al analizar el procedimiento que siguió el operador de justicia tradicional en el caso en mención, se evidencia la prevalencia de un orden jurídico positivista que no articula a las formas de organización social de los waorani ${ }^{12}$, que aporte al desarrollo de una resolución del caso pertinente, buscando que los hechos no queden en la impunidad, tal como sucedió en el 2003, cuando a pesar de la evidencia de una masacre no existió un juicio. Por ello, es necesario la construcción de un espacio de diálogo activo entre la justicia ordinaria y la indígena, no sólo de la etnia Waorani sino de los demás pueblos indígenas, para analizar este tipo de casos y así se impulse una discusión abierta a fin de desarrollar los argumentos con los que se logre construir sentencias, que respondan tanto a la gravedad de los hechos como al contexto social y cultural que motivó su cometimiento. Por lo anterior, es importante que se consideren las particularidades culturales de los actores involucrados y se establezcan sanciones que consideren ese contexto.

En relación al caso que presenta este trabajo, también es oportuno dejar abiertas algunas interrogantes para un trabajo de profundización posterior. Una de ellas es, cuestionar si el abordaje que hizo la justicia ordinaria sobre el caso en mención, es el inicio de un proceso para interculturalizar el derecho y su ejercicio, o si más bien, es un retorno al multiculturalismo, en donde el reconocimiento es simplemente funcional o aditivo en función de mantener el sistema nacional establecido. Limitarse a ciertos elementos de la cultura Waorani, resultará siendo conveniente para la justicia positivista imperante, ya que ello no afectará el statu quo del funcionamiento del sistema de justicia ordinario. Por ello, la noción de integrar la justicia indígena a la ordinaria tiene el riesgo de que no se repiense el sistema de justicia vigente ${ }^{13}$, y solamente se adicionen elementos del derecho indígena, que corresponden a soluciones jurídicas de orden temporal que no cambian el sistema de derecho positivo.

A pesar de lo anterior, la consideración de esos sistemas judiciales no debe hacerse integrando algunos de sus elementos, porque ello 
trae como riesgo que se mantenga el patrón que estaba presente también en la Constitución de 1998, es decir, un patrón neoliberal que subordinaba las culturas originarias a los sistemas establecidos en el Estado, entre ellos, el de la justicia. Es muy clara la necesidad de repensar la "estructura y sistema monocultural y uninacional" (Walsh, 2012), a partir de la construcción de la interculturalidad. Es justamente en este aspecto, en el cual es preciso puntualizar que la coexistencia de sistemas jurídicos diversos, en un Estado plurinacional e intercultural, no se da en un marco de igualdad y equidad, porque como indicamos anteriormente, los espacios en los que han interactuado el sistema de justicia ordinario con los indígenas ha sido en un espacio de subordinación donde ha prevalecido el sistema ordinario positivista.

Esta expresión de conflicto de poder, donde el Estado busca mantener su hegemonía, evidencia las estructuras del Estado y las actuaciones por mantener el orden imperante más allá de postulados constitucionales, dejando en una condición de sometimiento a las instituciones de los pueblos y nacionalidades indígenas. Díaz Polanco (1981), hace un análisis certero acerca de la hegemonía, subordinación y pueblos indígenas en los Estados latinoamericanos, donde su funcionamiento ha sido dirigido por élites que han invisibilizado a los pueblos y nacionalidades indígenas hasta que, en un determinado momento, a partir de luchas sociales y políticas, estos logran ser reconocidos, pero no de manera plena.

Es en los últimos quince años que los gobiernos de la región han legislado respecto a ese reconocimiento a la diversidad, pero no se han desarrollado reales políticas públicas que lleven a un ejercicio efectivo del mismo. Con lo anterior, los Estados siguen ejerciendo el poder, y a través de él, se mantiene una subordinación de los pueblos y nacionalidades indígenas. Además, se conservan las estructuras hegemónicas que reproducen acciones, no de invisibilización sino de marginalización de los temas indígenas. Para puntualizar, el reconocimiento a realizarse debe ser basado en usos y costumbres de los pueblos indígenas, pues el derecho consuetudinario de estos colectivos gira alrededor de estas prácticas, mientras que el derecho positivo se basa en jurisprudencia y legislación. Al respecto, Bartolomé (1997) manifiesta que las costumbres son la expresión de "sistemas políticos propios, históricamente constituidos y tan legítimos como los estatales". Así, esos sistemas políticos tienen sus instituciones y formas de funcionamiento propios, que implican reconocimiento, orden social interno, simbolismo y códigos internos que lo sustentan.

Por lo anterior, se hace necesario que el pluralismo jurídico se ejerza no solo reconociéndolo, sino repensando las estructuras del sistema de justicia ordinario para construirlo con bases que permitan el ejercicio de la plurinacionalidad e interculturalidad. Se hace inevitable alimentarse de experiencias de otros países, en donde se ha efectivizado un espacio de diálogo intercultural que permite el tratamiento de casos relacionados con pueblos indígenas, impidiendo que diferentes hechos queden en la impunidad. Boaventura de Sousa Santos (2009), señala que las diferencias entre culturas que comparten un mismo espacio territorial, requieren de "instituciones apropiadas", mientras que las semejanzas que existan en las culturas exigen "instituciones compartidas". De esta manera, una nueva perspectiva en la que se establezca el derecho de dialogar en formas iguales entre los sistemas de justicia indígena y el sistema positivista, es ineludible. 


\subsection{Límites de la justicia ordinaria}

Como se señaló, la justicia ordinaria en su concepción positivista, impide el ejercicio de dos principios constitucionales, la plurinacionalidad y la interculturalidad. Por una parte, el de plurinacionalidad, es decir, el ejercicio del reconocimiento a la diversidad cultural y el derecho a la consideración de dicha diversidad en las estructuras del Estado, y, por otro, la interculturalidad como un ejercicio de relacionamiento entre la diversidad cultural, basado en el respeto e igualdad ante el Estado.

Uno de los limitantes de la justicia es el reducido espacio para incorporar la interculturalidad; por lo que se hace necesario hacer un ejercicio de justicia "inclusiva, respetuosa y transparente" (Matos, 2010), donde se reconozcan las normas y sistemas de justicia, es decir, se reconozca el pluralismo jurídico existente. Como lo señala García (2010), en los casos que han sido conocidos por la Corte Constitucional, sobre derechos de los pueblos y nacionalidades indígenas, "los magistrados prefirieron hacer un análisis de legalidad antes que un análisis de interpretación constitucional" (Ídem: 12), lo que ha limitado la interpretación intercultural del derecho, impidiendo un avance en el pluralismo jurídico.

Es en este ámbito donde se presentan las tensiones, fundamentalmente por el conflicto que se genera al cuestionar las estructuras del Estado, y al plantear la necesidad de estructurarlas con una visión que ejerza los principios constitucionales. De esta manera, para abrir las puertas a un entendimiento intercultural se requiere de elementos que permitan a esa justicia positivista comprender esas pluralidades culturales existentes en un territorio. Uno de esos elementos viene de la mano de las ciencias sociales, principalmente de la antropología, que tiene la capacidad de ilustrar a los operadores de justicia sobre las particularidades culturales de las nacionalidades y pueblos originarios, respecto a los temas que son considerados en procesos judiciales, a través de los peritajes antropológicos.

Así, se abre la siguiente inquietud, ¿son los peritajes antropológicos la respuesta para la implementación de una justicia intercultural? $Y$ en este sentido, es preciso señalar que los peritajes, son medios probatorios que emplea el sistema judicial con la finalidad aportar a la convicción judicial sobre las responsabilidades en torno al caso para el cual son solicitados. En el caso de los peritajes antropológicos, estos se convierten en una prueba que brinda información al juzgador sobre la importancia de la diferencia cultural en el entendimiento de un caso.

El fin de los peritajes antropológicos es identificar el contexto cultural de la persona procesada, enmarcándola dentro de las tradiciones y prácticas como pueblo o nacionalidad indígena; así, se identifica el universo simbólico y social que intervino en la actuación del individuo. Matos (2010) señala que el peritaje antropológico expone si hay un error de comprensión culturalmente condicionado sobre la normativa nacional vigente, y ella da lugar a la actuación ilícita del inculpado. El peritaje antropológico es una herramienta para que los operadores de justicia lleguen a tener un enfoque intercultural, por la heterogeneidad cultural existente, donde se da una convivencia entre distintas culturas, a pesar de que esta sea inequitativa, excluyente y demás características expuestas en los primeros capítulos de este trabajo. De esta manera se abre espacio para un diálogo respetuoso entre sistemas jurídicos distintos. 
Los peritajes antropológicos apoyan en la construcción de un escenario para la relación entre el Estado y los pueblos indígenas (Sánchez Botero, 1992); desarrollan las condiciones de equidad para los pueblos y nacionalidades indígenas, pues, a través de ellos, se reconocen las prácticas culturales, las cuales se desarrollan en un contexto social, histórico, económico y político interno, y que pudieran ser consideradas como actos delictivos por el derecho positivo. La información que desarroIla un peritaje antropológico abre la posibilidad de entendimiento a los operadores de justicia, como un espacio de diálogo (Valladares, 2011; Matos, 2010).

La importancia radica en que la interpretación de la Corte Constitucional a través de la Sentencia № 004-14-SCN-CC. Caso № 0072-14-CN establece la aplicación del Código Penal, considerando el contexto del principio de diversidad étnica y cultural, que no aborda el aspecto de la interculturalidad, sino solamente se limita a aspectos de forma, como son las consideraciones en torno a la lengua, a los aspectos culturales, el entorno social, y otros detallados en el Convenio 169 de la OIT. Además, establece la competencia en los operadores de justicia del análisis de las "circunstancias sociológicas de los pueblos que se encuentran en el conflicto penal y las circunstancias del hecho que se investiga", con las consideraciones necesarias del principio de diversidad cultural garantizado en la Constitución. Sin embargo, de lo anterior, la interpretación intercultural del derecho aún no está resuelta.

Es importante plantear que el principio de interculturalidad debe debatirse no solamente en el ámbito de la justicia, sino de manera integral. Aquí cabe dejar varias interrogantes que solo podrán ser resueltas con una ruptura en el ordenamiento jurídico positivista: ¿los waorani acusados del delito de homicidio contra pueblos en aislamiento deben o no ser detenidos? ¿Ser waorani significa estar por fuera del sistema de justicia?, si es así, ¿dónde queda el pluralismo jurídico?, o es que este ¿va a ser entendido como un sistema de beneficios? Lo anterior se configura en una discusión necesaria que deberá ampliarse, previo a la resolución del caso, con la finalidad de que la decisión tomada por los operadores de justicia responda al contexto de principios constitucionales, pero sobre todo a las garantías de derechos de todos los habitantes del país que involucra a los pueblos en aislamiento.

\section{Conclusiones}

De acuerdo al análisis realizado, podemos establecer que, en el ámbito de la justicia intercultural en Ecuador, no se identifican avances, más bien a través de Sentencias de la Corte Constitucional se ha limitado la competencia para sancionar casos relacionados con atentados a la vida de las personas al Sistema de Derecho Penal Ordinario, excluyendo de su tratamiento a las autoridades indígenas, y subordinando a los sistemas de justicia indígena al sistema de justicia ordinario. De esta manera, el espacio de diálogo intercultural que aportaría a la resolución de casos complejos que tengan relación con pueblos y nacionalidades indígenas se ve limitado y requiere de procesos de capacitación en temas de justicia intercultural a los operadores de justicia.

Se ha visto a la coordinación de la justicia ordinaria y de la justicia indígena como un espacio de diálogo intercultural, pero este se ha limitado 
a incorporar ciertos elementos de las culturas indígenas en el tratamiento judicial, sin reconstruir el sistema de justicia a través de espacios que permitan un verdadero diálogo entre las culturas y la toma de decisiones de carácter judicial. Es necesario que ese diálogo intercultural se realice de forma igualitaria entre el derecho ordinario y los sistemas indígenas, sin una prevalencia del uno sobre el otro. Es importante no solo reconocer la diversidad cultural existente en el país, sino construir espacios de diálogo y relacionamiento activo, tendientes al ejercicio de los derechos de los pueblos indígenas y de la sociedad en su conjunto. El reconocimiento constitucional a la diversidad requiere desarrollar derechos para dar contenido al enunciado constitucional.

Desde la práctica profesional de la antropología se puede aportar al ejercicio de la interculturalidad en el ámbito de la justicia, brindando elementos al derecho positivo, a través de peritajes, estudios especializados y análisis sociales y culturales, que abran la puerta a la comprensión de las particularidades culturales involucradas en un proceso judicial determinado. La práctica profesional de la antropología no tiene como función realizar una traducción cultural, sino interpretar las prácticas culturales cuestionadas en un determinado caso, para conocer los esquemas y referentes cognitivos que estuvieron involucrados en el cometimiento de un acto, el cual fue judicializado por la justicia ordinaria por ser contrario a las leyes vigentes.

La plurinacionalidad requiere de regímenes integrales de vida social, desarrollados y el ejer- cicio de la interculturalidad abre la posibilidad para que la justicia ordinaria sea confrontada con otros saberes, en el marco de unos principios constitucionales claramente establecidos. La interculturalidad rompe con la relación de fuerzas y subordinación de los sistemas judiciales al derecho positivista, al permitir las relaciones entre sistemas de derecho ordinario y sistemas jurídicos indígenas.

La interculturalidad es un proceso en construcción, que va más allá de principios establecidos en la Constitución y que es preciso aportar a su establecimiento, principalmente con el desarrollo de políticas públicas.

El Estado ecuatoriano no ha tenido la capacidad de garantizar la vida de un grupo en extremo vulnerable. Más allá del proceso judicial, existen víctimas, y en función de ello es que debe garantizar, a través de un proceso judicial efectivo, que un hecho similar no vuelva a ocurrir. Un estado de impunidad puede desatar otras acciones, tal como ha venido sucediendo. La impunidad de los hechos del 2003 dejó la puerta abierta para una nueva masacre, como la ocurrida en el 2013. Si nuevamente el sistema de justicia no resuelve de manera adecuada, se corre el riesgo de que se produzca una nueva matanza, en una zona caracterizada por una diversidad de actores presentes, intereses económicos, pueblos indígenas de reciente contacto y sin contacto, que configuran una conflictividad sobre la cual el Estado debe actuar para garantizar la vida de los pueblos más vulnerables.- 


\section{Notas}

${ }^{1}$ Es a partir de la década de los noventa con la lucha del movimiento indígena en varios países andinos, y la guerra en Chiapas para el caso mexicano, que se visibilizan los pueblos y nacionalidades. A pesar de ello, esa visibilización no significó que se desarrolle una política pública que atienda las demandas planteadas desde estos sectores.

${ }^{2}$ Los waorani son un pueblo amazónico de reciente contacto que habitan en el Ecuador.

${ }^{3}$ Se enunciará como Tagaeri - Taromenane a uno de los varios grupos familiares de pueblo en aislamiento que habita en la región del Yasuní.

${ }^{4}$ Pueblos indígenas aislados (PIA) o en situación de aislamiento es la denominación que se da a aquellos grupos familiares que guardan sus formas de vida tradicionales y que han optado por mantenerse al margen de las sociedades nacionales (Rivas, 2003). Naciones Unidas define a los pueblos indígenas en aislamiento como "pueblos o segmentos de pueblos indígenas que no mantienen contactos regulares con la población mayoritaria y que además suelen rehuir todo tipo de contacto con personas ajenas a su grupo (Oficina del Alto Comisionado de Naciones Unidas para Derechos Humanos, 2012: 8).

${ }^{5}$ Establecida en el año 1999 a través de Decreto Ejecutivo, tiene como finalidad excluir el territorio de cualquier actividad de índole extractiva que pueda afectar las condiciones ambientales y culturales.

${ }^{6}$ Organización de la Nacionalidad Huaorani del Ecuador.

${ }^{7}$ El Agente Fiscal de la Provincia de Pastaza, Dr. Marco Vargas inició la indagación fiscal № 347-2003. De acuerdo al fiscal "dirigentes de la ONHAE, han presionado para que la justicia común no continúe investigando, por lo que, debido a esto y la dificultad de por lo menos conocer los nombres de los fallecidos y los posibles participantes en el delito por falta de colaboración, se ha estancado la indagación." Oficio № 257MFD-PZ de diciembre 12 de 2006 dirigido a la Secretaria general del Ministerio Público" (INREDH, 2009).

${ }^{8}$ Algunos de los atacantes que participaron en la masacre tenían lazos familiares directos con los dirigentes waorani de la época.

${ }^{9}$ La Constitución de 1998 no preveía procesos de declinación de competencia de la justicia ordinaria en favor de la indígena.

${ }^{10}$ Corte Constitucional del Ecuador. Sentencia № SCC 004-14-SNCCC, Caso № 0072-14-CN de 6 de agosto 2014.

${ }^{11}$ García (2010), expone la existencia de al menos siete casos relacionados con derechos de pueblos indígenas, que han sido tratados por la Corte Constitucional, durante el periodo 1998-2008. Dos de ellos tienen similar nivel de complejidad, que fueron los denominados "La Cocha 1" y "La Cocha 2", enmarcados dentro del ámbito penal, y relacionados con hechos de asesinato.

${ }^{12}$ Como se señaló anteriormente, se requeriría identificar la existencia de un sistema de justicia waorani, o la forma social y cultural de ordenamiento interno de los waorani.

${ }^{13}$ Comentario personal de la Dra. Catherine Walsh, 2 de marzo de 2015 . 


\section{Referencias bibliográficas}

Aguirre, M. (2007). ¡A quién le importan esas vidas!: un reportaje sobre la tala ilegal en el Parque Nacional Yasuní. Quito: CICAME.

Ávila, L. (2011). Barreras ideológicas para la valoración judicial de los peritajes antropológicos. Revista Crítica Jurídica, № 31: 15 - 37.

Bartolomé, M. (1997). Gente de costumbres y gente de razón. Las identidades étnicas en México. México: Siglo XXI Editores.

Cervone, E. (2009). Los desafíos del multiculturalismo. En: Repensando los Movimientos Indígenas. Quito: Facultad Latinoamericana de Ciencias Sociales, Ministerio de Cultura.

Chávez, G. (2003). "Muerte Tagaeri-Taromenane: justicia occidental o tradicional". Íconos: revista de ciencias sociales. El ataque de los tagaeri. № 17, septiembre, pp. $31-36$.

Clavero, B. (2008). Geografía jurídica de América Latina. Pueblos Indígenas entre constituciones mestizas. México: Siglo XXI Editores.

Corte Constitucional del Ecuador. Sentencia a la Acción Extraordinaria de Protección, Código 0731-10-EP de 30 de julio de 2014.

Corte Constitucional del Ecuador. Sentencia № 004-14-SNCCC, Caso № 0072-14-CN de 6 de agosto del 2014.

Díaz Polanco, H. (1981). "Etnia, clase y cuestión nacional". Cuadernos Políticos, № 30, octubre-diciembre, pp. 53-65. (2006). Elogio de la diversidad. Globalización, multiculturalismo y etnofagia. México: Siglo XXI.

García, D. (2007). "Pluralismo Jurídico y Peritaje Antropológico: su incidencia en la Educación y legislación Ecuatoriana”. En II Congreso Ecuatoriano de Antropología y Arqueología Balance de la última década: Aportes, retos y nuevos temas. Tomo II. Quito: Abya Yala y Banco Mundial.

García, F. (2010). "Retos de la diversidad: el reconocimiento y aplicación de los sistemas de derecho indígena ecuatoriano". Íconos: Revista de ciencias sociales. № 38, septiembre, pp. 9 - 16.

Gazohui, R. (2008). Derechos colectivos y multiculturalidad. En: Alba. O. \& Castro, S.R.. (Coords.), Pluralismo Jurídico e Interculturalidad. La Paz: Comisión de Justicia de la Asamblea Constituyente, pp. 239-258.

Grijalva, A. (2008). "El Estado Plurinacional e Intercultural en la Constitución Ecuatoriana del 2008”. Revista Ecuador Debate, № 75. Quito: Centro Andino de Acción Popular. pp. 49-62.

INREDH. (2009). Derechos de los pueblos indígenas en Ecuador Audiencia -137 Período ordinario de sesiones. Comunicación de 3 de noviembre dirigida a Santiago Cantón, Secretario Ejecutivo de la Comisión Interamericana de Derechos Humanos.

Juzgado de Garantías Penales de Orellana. Proceso Penal № 223-2013.

Masapanta, C. (2009). "El derecho indígena en el contexto constitucional ecuatoriano: entre la exigibilidad de derechos y el reconocimiento". En Derechos ancestrales: justicia en contextos plurinacionales. Quito: Ministerio de Justicia y Derechos Humanos. pp. $409-450$.

Mato, J. (2010). "¿La interculturalidad se abre paso en las Cortes Superiores de Justicia de la Amazonía peruana?" Ponencia presentada en el VII Congreso Internacional de la Red Latinoamericana de Antropología Jurídica. Lima, 2 al 6 de agosto.

Narváez, R. (2013). Informe Diligencia de reconocimiento del lugar de los hechos, Indagación Fiscal Nro. 22020181304001 por Presunto Delito de Genocidio - Etnocidio. Fiscalía General del Estado, Comisión de la Verdad y Derechos Humanos. Informe técnico sin publicar. Quito. Ecuador.

Rivas, A. (2003). "Sistema mundial y pueblos indígenas en la Amazonía: a propósito del ataque a los tagaeri (Coyuntura)". Íconos: revista de ciencias sociales. El ataque de los tagaeri, № 17, septiembre, pp. 21-30.

Sánchez Botero, E. (1992). "Peritazgo antropológico. Una forma de conocimiento. El otro Derecho". ILSA Sociología y Ciencias políticas, № 2 .

(2010). Justicia y pueblos indígenas de Colombia. Bogotá: Universidad Nacional de Colombia.

De Sousa Santos, B. (2009). "Las paradojas de nuestro tiempo y la Plurinacionalidad" En Plurinacionalidad: Democracia en la diversidad. Quito: Editorial Abya Yala, pp. 24-25.

Stavenhagen, R. (2000a). Conflictos étnicos y estado nacional. México: Siglo XXI editores.

(2000b). "Derechos humanos y ciudadanía multicultural: Los pueblos indígenas" En Jean François Prud'homme (Comp.). Demócratas, Liberales y republicanos, México: El Colegio de México. pp. $79-89$.

Valladares, L. (2011). "El peritaje Antropológico. Los retos del entendimiento intercultural”. En Baeza Espejel, G., Gómez, G. \& Ramón, N.E. Pueblos indígenas. Debates y Perspectivas. México: Edit. Programa. Universidad Autónoma de México.

(2012). "La importancia del peritaje cultural: avances, retos y acciones del Colegio d Etnólogos y Antropólogos Sociales, A.C. (CEAS) para la certificación de peritos". En Peritaje antropológico en México: reflexiones teórico metodológicas y experiencias. México: Boletín Colegio de Etnólogos y Antropólogos Sociales.

Vargas, M. \& Gálvez, M. (2007). Los Últimos Guerreros de la Selva. Puyo: Casa de la Cultura de Pastaza.

Velasco Gómez, A. (2004). "Multiculturalismo, Nación y Federalismo". Revista Mexicana de Ciencias Políticas y Sociales. Mayo - agosto, año/vol. XLVII, № 191, pp.68-85.

Walsh, C. (2012). "Interculturalidad crítica y pluralismo jurídico: Reflexiones en torno a Brasil y Ecuador". En Interculturalidad y (de) colonialiad. Ensayos desde el Abya Yala. Quito: Abya Yala, Instituto de Culturas Indígenas ARY. 
ve $v y, v, v y, w, k y$

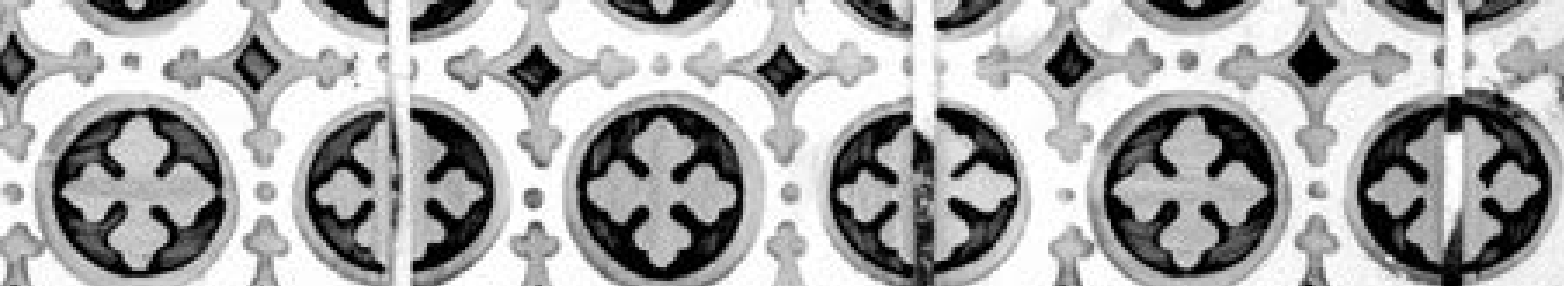
- 04

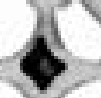

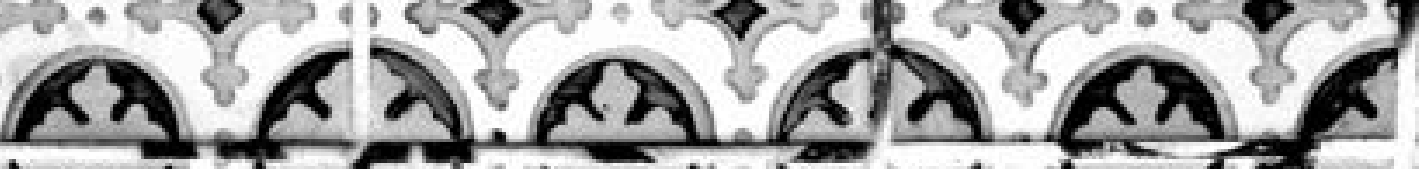

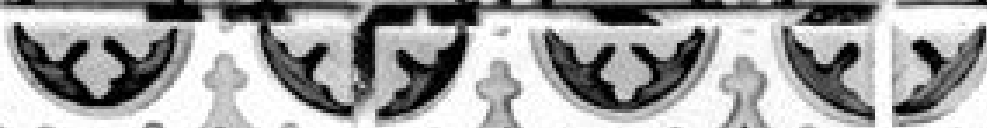
$430 \rightarrow$

(3) $<3$

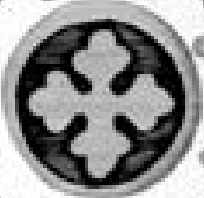

$\rightarrow+$
45
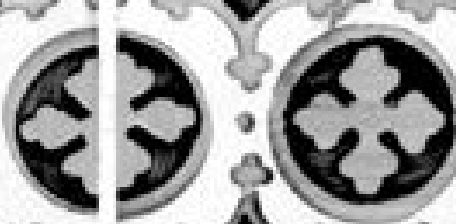

एर
(1).
个

की 00
Ca.chia (ख) kiv 4

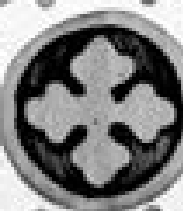

(3)

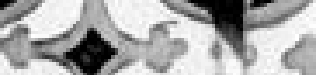

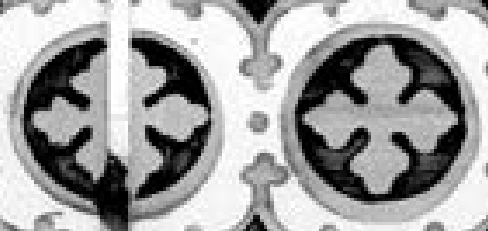

0
A

(

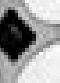

\section{A. 6}

(n)

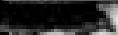
A ज) $अ$

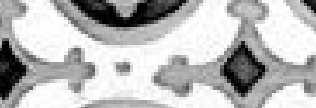

\section{$\forall Y$ एर}

(4)

8
32.0

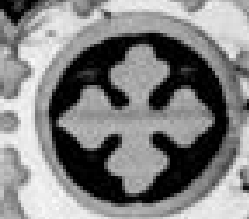

$2>S$ \& $P$

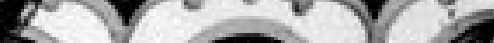

47 as
4

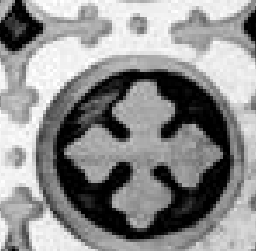

$405 \cdot 00$

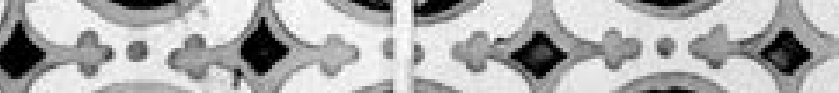

$3(y, 3): 8$

\section{(v) $R=$}

W $Y$

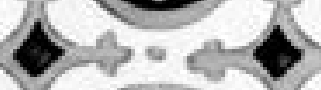

$\alpha \lambda$

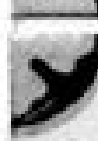

\section{(}

